Frequency Domain Subspace Identification Using Nuclear Norm Minimization and Hankel Matrix Realizations

Journal Article

Author(s):

Smith, Roy (i)

Publication date:

2014-11

Permanent link:

https://doi.org/10.3929/ethz-b-000093277

Rights / license:

In Copyright - Non-Commercial Use Permitted

Originally published in:

IEEE Transactions on Automatic Control 59(11), https://doi.org/10.1109/TAC.2014.2351731 


\title{
Frequency Domain Subspace Identification using Nuclear Norm Minimization and Hankel Matrix Realizations
}

\author{
Roy S. Smith, Fellow, IEEE
}

\begin{abstract}
Subspace identification techniques have gained widespread acceptance as a method of obtaining a low-order model from data. These are based on using the singular-value decomposition as a means of estimating the underlying system order and extracting a basis for the extended observability space. In the presence of noise rank determination becomes difficult and the low rank estimates lose the structure required for exact realizability. Furthermore the noise corrupts the singular values in a manner that is inconsistent with physical noise processes. These problems are addressed by an optimization based approach using a nuclear norm minimization objective. By using Hankel matrices as the underlying data structure exact realizability of the low rank system models is maintained. Noise in the data enters the formulation linearly, allowing for the inclusion of more realistic noise weightings. A cumulative spectral weight is presented and shown to be useful in estimating models from data corrupted via noise. A numerical example illustrates the characteristics of the problem.
\end{abstract}

Index Terms-System identification; State-space methods; Optimization methods; Pareto Optimization; Linear algebra.

\section{INTRODUCTION}

$\mathbf{S}$ UBSPACE identification methods directly address the problem of obtaining a state-space realization of a loworder system from noisy data. These methods have been developed over the last 20 or more years and there is an extensive literature on the topic. See for example [1]-[5]. Both frequency- and time-domain formulations are commonly used. The work presented here will emphasize frequency domain methods as these usually lead to lower data dimension matrix problems better suited to the optimization methods to be employed. However the underlying ideas are also be applicable to time-domain methods.

The basis for subspace identification methods is the construction-from data-of matrices with range spaces matching the extended observability subspace of an underlying low order system. From this extended observability space one can factor the output matrix, $C$, and the state transition matrix, $A$, of the underlying system. Determining the input matrix, $B$, and the feedthrough matrix, $D$, is then a linear problem, typically solved via least-squares methods. See [6] for much more detail on the realization theory and methods that arise in this class of problems. Frequency domain subspace identification

Manuscript received 16 November 2012; revised sometime in 2013; received in final form 15 January 2014. Recommended by Associate Editor:?

R. Smith is with the Automatic Control Laboratory at the Swiss Federal Institute of Technology, ETH, Zürich. rsmithecontrol.ee.ethz.ch methods were developed and analyzed by McKelvey et al. [7], and are used here as a starting point for our optimization based methods. See also [8] for an alternative formulation leading to a similar subspace determination problem.

The extended observability spaces generated by linear timeinvariant systems have a specific structure. In the uniformly frequency spacing case the matrices considered have Hankel structure; and in the general case a similarly parameterized matrix range is required. With the given structure (which for simplicity we describe as a "shift structure") the $C$ and $A$ matrices can be factored directly. Ideally the data matrices have both low rank and shift structure. Unfortunately both properties may be lost in the noisy data case.

The most common method of determining the range space of the data matrix is the singular value decomposition (SVD). In low noise cases an analysis of the dominant singular values leads to reasonable estimates of the dimension of the underlying extended observability space. Unfortunately truncating the matrix by removing the small singular values destroys the shift structure required for reconstruction. One typically then estimates $C$ and $A$ from the chosen range-space basis via least-squares or total-least-squares methods. The quality of the resulting estimate depends on the underlying spaces. When the noise is significant it is often difficult to determine a suitable rank for the range space. Furthermore, the noise on individual measurements affects the data matrix and the singular values in a highly nonlinear way. These difficulties are discussed in greater detail in Section II-C.

Several researchers have studied a variety of ways of overcoming the problems with noisy data subspace identification. One approach for improving the quality of the estimate for $C$ and $A$ from the range space basis uses total least-squares (TLS) [9]. Accounting for the explicit algebraic constraints in a noisy extended range-space basis leads to constrained total least-squares (CTLS) methods [10]. A similarly motivated approach uses block row weighting in the singular value calculation [11].

Ideally one would want a method for low rank matrix approximation that preserves the shift structure of the range space. Formulating this as a minimum rank optimization problems gives an NP-hard problem. Nevertheless, significant progress has been made on matrix rank minimization problems in the last decade. The most promising approach involves minimizing the sum of the singular values of the matrix; a quantity also referred to as the nuclear norm, or the trace-norm. 
Early work by Fazel et al. [12] illustrated this on a simple model identification problem. However the use of a tracenorm optimization objective in the closely related problem of model reduction was previously investigated by Beck et al. [13]. Recht et al. [14] have only recently determined why this heuristic works well as it does.

This approach is now being actively developed for application to system identification. For example, iteratively reweighted nuclear norm methods are considered in [15]. Hjalmarsson et al. [16] use a nuclear norm relaxation in an ARX identification problem. Algorithmic methods, tailored to system identification constraints, are presented in [17], [18]. Our work is closer to the approaches taken by Liu and Vanderberghe [19] and Grossman et al. [20]. The later paper also addresses the problem of missing data. Similar work, using a blind separation approach is given in [21]. All of these approaches focus on the time-domain case and most also use the nuclear norm for a regularization term in the minimization problem.

Our approach involves the use of the nuclear norm minimization problem as a means of investigating the Pareto optimal trade-off between the model order (or at least its nuclear norm surrogate) and size of the residual. We pose the problem in terms of minimizing the nuclear norm of matrixvalued function, with the closeness of the fit to the data being enforced by constraints. This is equivalent to a regularizationbased optimization (see [22]), but is more direct in generating the trade-off curve of interest.

We develop a parametrization that satisfies the shift structure requirements for exact state-space reconstruction and is also linear in the residual error. This makes it possible to apply a variety of residual error objectives directly. In particular, we introduce a weighted cumulative spectral constraint to ensure that error residuals also possess realistic noise-like properties. This is motivated by the set based white noise descriptions introduced by Paganini [23] for use in robust control. This form of residual characterization is useful in countering the bias effect due to nuclear norm minimization (or regularization).

Earlier work by the author in [24] applied nuclear norm minimization methods using the cumulative spectral constraint to the uniform frequency sample case. The current paper extends this work and provides a complete parametrization that also addresses the nonuniformly spaced data case. Additional details are also provided on exact realization methods.

\section{A. Notation}

We consider models parametrized as discrete-time statespace representations,

$$
\begin{aligned}
x(k+1) & =A x(k)+B u(k), & A \in \mathcal{R}^{n_{x} \times n_{x}} \\
y(k) & =C x(k)+D u(k), & D \in \mathcal{R}^{n_{y} \times n_{u}} .
\end{aligned}
$$

If the above system is denoted by $G$ then a frequency response calculation of $G$ at $M$ frequencies, $\omega_{n}, n=1, \ldots, M$, can be considered as a three dimensional vector, with frequency as the third dimension. For simplicity we will abbreviate the frequency response as $G \in \mathcal{C}^{n_{y} \times n_{u} \times M}$. The notation $g \in \mathcal{R}^{n_{y} \times n_{u} \times L}$ is used for $L$ corresponding impulse response matrices. Mathematical operators such as $\operatorname{diag}()$, are interpreted as block operations in this notational context.

Given $x \in \mathcal{R}^{M}$, representing $M$ equi-spaced samples of a scalar time-domain signal, we use $X=\mathcal{F}(x) \in \mathcal{C}^{M}$, to denote the $M$-length Discrete Fourier Transform of $x$. Similarly, $\mathcal{F}^{-1}(X)$ denotes the corresponding inverse transform. This notation is extended to the MIMO case for $x \in \mathcal{R}^{n_{y} \times n_{u} \times M}$ with $X=\mathcal{F}(x)$, where $X \in \mathcal{C}^{n_{y} \times n_{u} \times M}$ is given by $X_{i, j}=\mathcal{F}\left(x_{i, j}\right), i=1, \ldots, n_{y}$ and $j=1, \ldots, n_{u}$.

The transpose of a matrix $X$, is denoted by $X^{T}$, the conjugate is $\bar{X}$, the conjugate transpose is $X^{*}$ and the null space is $\mathcal{N}(X)$. The nuclear norm, or sum of the singular values, is $\|X\|_{*}$. Unit vectors, with a 1 in the $j$ th component are denoted by $e_{j}$. Kronecker products will be used to simplify the notation for block structured matrices. Of particular use is the product identity,

$$
(X \otimes Y)(W \otimes Z)=X W \otimes Y Z .
$$

To further simplify the notation for block structured matrices we use,

$$
[f(X, Y, \cdots, i, j)] \begin{aligned}
& i=1, \ldots, p \\
& j=1, \ldots, q
\end{aligned},
$$

to denote matrices where the $i, j$ block is constructed as a function, $f(X, Y, \ldots, i, j)$ of matrices $X, Y, \ldots$, and the indices $i$ and $j$. The integers $p$ and $q$ above denote the number of block rows and block columns respectively. Note that Hankel matrices have the property that the index dependence of $f(X, Y, \ldots, i, j)$ can be expressed as function of $i+j$.

We define a linear function, $\mathcal{H}(x)$, which maps a (block) vector of length $p+q-1$ to a (block) Hankel matrix of size $p \times q$ via,

$$
\mathcal{H}(x)=\left[\begin{array}{cccc}
x_{1} & x_{2} & \cdots & x_{q} \\
x_{2} & x_{3} & & x_{q+1} \\
\vdots & & \ddots & \vdots \\
x_{p} & x_{p+1} & \cdots & x_{p+q-1}
\end{array}\right]
$$

\section{FREQUENCY DOMAIN SUBSPACE IDENTIFICATION}

\section{A. Problem context}

Frequency domain subspace identification, with uniformly spaced frequency samples, is described here to both introduce the problem setting that we consider and to indicate some of the shortcomings with the standard approach.

We consider the problem of identifying a MIMO state-space realization,

$$
G\left(\mathrm{e}^{j w}\right)=C\left(\mathrm{e}^{j \omega} I-A\right)^{-1} B+D,
$$

from a set of uniformly spaced frequency domain matrixvalued data,

$$
G_{n} \in \mathcal{C}^{n_{y} \times n_{u}}, \quad \omega_{n}=\frac{\pi n}{M}, \quad n=0, \ldots, M .
$$

The restriction to uniformly spaced estimates provides a simplified setting for the introduction of the problem and leads to a 
simplified algorithm. The more general non-uniformly spaced case is treated in Section V.

In practice our estimates are generated from preprocessed noisy data, and in this context the user's a priori assumption is typically that the data were generated by a linear low dimensional system and corrupted by noise in the measurement process. We are therefore interested in finding a realization, $\hat{G}\left(\mathrm{e}^{j w}\right)$, consistent with the observation $G$, and noise $W$, such that:

$$
G_{n}\left(\mathrm{e}^{j \omega_{n}}\right)=\hat{G}\left(\mathrm{e}^{j \omega_{n}}\right)+W_{n}, \quad n=0, \ldots, M,
$$

or, equivalently, $G=\hat{G}+W$. Furthermore we are interested in the estimated model $\hat{G}\left(\mathrm{e}^{j \omega}\right)$, being of low order and $W_{n}$ being small in norm and resembling a realization of a noise process.

The approach taken in this paper differs from the more standard identification formulation; the definition of the best estimated model, $\hat{G}\left(\mathrm{e}^{j \omega}\right)$, is driven by the application at hand and the underlying biases of the user. The focus of this paper is to provide the user with tools to investigate the trade-off between model order, the size of the consistent noise, $W_{n}$, and the "noise-like" properties of $W_{n}$.

\section{B. Singular-value based subspace identification}

The commonly used algorithms for both time- and frequency-domain subspace identification are based on the singular-value decomposition (SVD). The frequency-domain approach was developed by McKelvey et al. [7] and we will use their basic outline in the description here. Given $M+1$ estimates, uniformly spaced on positive frequencies, (as in (1)), one first extends the data to negative frequencies,

$$
G_{n}=\bar{G}_{2 M-n}, \quad n=M+1, \ldots, 2 M-1,
$$

with $\bar{G}_{n}$ denoting the complex conjugate of $G_{n}$. An inverse Fourier transform of these estimates is calculated, giving $h \in$ $\mathcal{R}^{n_{y} \times n_{u} \times 2 M}$ as,

$$
h_{k}=\frac{1}{2 M} \sum_{n=0}^{2 M-1} G_{n} \mathrm{e}^{j 2 \pi n k / 2 M}, \quad k=0, \ldots, 2 M-1 .
$$

One now forms the block Hankel matrix, given here as blocksquare for simplicity,

$$
H=\mathcal{H}(h) \in \mathcal{R}^{n_{y} M \times n_{u} M} .
$$

If the data, $G$, was generated, without noise, by an underlying minimal realization, $A, B$ and $C$, then $H$ can be factored as,

$$
\begin{aligned}
& H= \\
& {\left[\begin{array}{c}
C \\
C A \\
\vdots \\
C A^{M-1}
\end{array}\right]\left(I-A^{2 M}\right)^{-1}\left[\begin{array}{llll}
B & A B & \cdots & A^{M-1} B
\end{array}\right]} \\
& =\mathcal{O}\left(I-A^{2 M}\right)^{-1} \mathcal{C},
\end{aligned}
$$

where $\mathcal{O}$ and $\mathcal{C}$ are the extended observability and controllability matrices respectively. In the following we will refer to a matrix having the form of $\mathcal{O}$ above as having "shift structure."
As $H$ has rank equal to $n_{x}$, an SVD of $H$ gives,

$$
H=\left[\begin{array}{ll}
U_{1} & U_{2}
\end{array}\right]\left[\begin{array}{cc}
\Sigma_{1} & 0 \\
0 & 0
\end{array}\right]\left[\begin{array}{c}
V_{1}^{T} \\
V_{2}^{T}
\end{array}\right]=U_{1} \Sigma_{1} V_{1}^{T} .
$$

Furthermore, as $U_{1}$ is a basis for $\mathcal{O}$, there exists an invertible matrix $T$ such that, $\mathcal{O}=U_{1} T^{-1}$. Factoring $U_{1}$ such that,

$$
U_{1}=\left[\begin{array}{c}
\hat{C} \\
\hat{C} \hat{A} \\
\vdots \\
\hat{C} \hat{A}^{M-1}
\end{array}\right],
$$

gives estimates, $\hat{C}$ and $\hat{A}$ related to the underlying system by, $\hat{C}=C T$, and $\hat{A}=T^{-1} A T$. With $\hat{C}$ and $\hat{A}$ given, determining $\hat{B}$ and $\hat{D}$ reduces to a linear fitting problem which is commonly solved via least-squares.

\section{Noisy data case}

The approach given in the previous section is exact-to within a similarity transform-for noise-free data and provides a strong motivation for applying a similar approach to the noisy data case. In this case the SVD in (4) instead becomes,

$$
\begin{aligned}
H=\mathcal{H}(h+w)=\left[\begin{array}{ll}
U_{1} & U_{2}
\end{array}\right] & {\left[\begin{array}{cc}
\Sigma_{1} & 0 \\
0 & \Sigma_{2}
\end{array}\right]\left[\begin{array}{l}
V_{1}^{T} \\
V_{2}^{T}
\end{array}\right] } \\
& =U_{1} \Sigma_{1} V_{1}^{T}+U_{2} \Sigma_{2} V_{2}^{T} .
\end{aligned}
$$

It is no longer possible to uniquely assign the contributions of the noise and underlying low-order system to the various singular values. The noise contribution, $\mathcal{H}(w)$, corrupts $U_{1}$, $\Sigma_{1}$ and $V_{1}$ in addition to generating the additional singular values and vectors in $\Sigma_{2}, U_{2}$ and $V_{2}$. A reasonable heuristic is to attribute the largest singular values to the underlying loworder system and the remaining-hopefully small-singular values to the noise. This then leads to the approximation

$$
\mathcal{H}(h) \approx U_{1} \Sigma_{1} V_{1}^{T},
$$

as a justification for using $U_{1}$ as an estimate of the range space of the underlying system's extended observability space.

The estimates $\hat{C}$ and $\hat{A}$ are based on partitions of $U_{1}$ in (5). The output matrix estimate is given by,

$$
\hat{C}=\left[\begin{array}{ll}
I_{n_{y}} & 0
\end{array}\right] U_{1},
$$

and the state-transition matrix is given as the least-square solution of,

$$
\left[\begin{array}{ll}
I_{n_{y}(M-1)} & 0
\end{array}\right] U_{1} \hat{A}=\left[\begin{array}{ll}
0 & I_{n_{y}(M-1)}
\end{array}\right] U_{1} .
$$

There are several difficulties that arise with SVD-based subspace identification. These are known and will only be summarized here. See [24] for more details.

a) Determining the appropriate rank of $H$ : This is typically done by selecting an index where the singular values drop significantly. Unfortunately it is not always straightforward. The addition of noise to the matrix $H$ makes all of the singular values non-zero and while the noise added to each element of $H$ is relatively small, the cumulative effect on the $n_{x}+1$ st singular value can be large. This effect is amplified by the Hankel structure of both $H$ and the added noise, as discussed 
in $c$ ) below. The example considered in Section VI illustrates a case where it is difficult to determine an appropriate rank from the singular values.

b) The lack of shift structure in the truncated SVD: Given a Hankel matrix, $H$, one can find matrices, $A, B$ and $C$ satisfying (3) and suitable methods for finding these matrices are given in Section IV. In general, if $H \in \mathcal{R}^{n_{y} M \times n_{u} M}$, the representation will be of order $2 M-1$. In this case the basis for the range space of $H$ in (6) is $U=\left[\begin{array}{ll}U_{1} & U_{2}\end{array}\right]$ and $U$ has the required shift structure. However $U_{1}$ alone does not generically have this shift structure and there is no reduced order $\hat{C}$ and $\hat{A}$ which would generate the required $U_{1}$. The least-squares estimate given in (8) suffers from the problem that errors occur in both the left and righthand factors. Total least-squares gives some improvement but does not account for the fact that for the majority of matrix components in (8) the errors in the left and righthand sides are actually equal.

c) The weighting of the effects of the noise: The contribution of the noise, $w=\mathcal{F}^{-1}(W)$ in (2), to the singular values of the Hankel matrix $H=\mathcal{H}(h+w)$ is not simple. The effect of $W$ on the individual blocks, $h_{i}$, of $\mathcal{H}\left(h_{i}\right)$ is linear (via the Fourier transform) but different blocks appear in $H$ a different number of times. For example, $h_{M}$ appears $M$ times, whereas $h_{1}$ and $h_{2 M-1}$ appear each only once. One of the consequences of this is that the variance of the effect of Hankel structured noise on the singular values appears to be larger than unstructured noise. If one were to weight the effect of individual noise on $h_{i}$, this weighting would have to be applied via a Hadamard multiplication. Multiplicatively weighted SVD problems will not correctly account for such noise.

\section{NUCLEAR NORM-BASED PROBLEM FORMULATION}

The subspace identification problem is reformulated in order to address each of the three problems outlined in the previous section. We consider the problem in terms of an optimization that attempts to find a noise signal, $W \in \mathcal{C}^{n_{y} \times n_{u} \times M}$, and an exact realization of a low order system that is consistent with the the observed data-in otherwords, satisfying (2). As neither the noise, nor the underlying system, is known there are many such consistent noise/model pairs. Our approach involves finding a selection of such noise/model pairs that lie on a Pareto optimal curve with respect to model order, and noise size.

The first step is to formulate a Hankel matrix as a function of both the data and an unknown noise, $W \in \mathcal{C}^{n_{y} \times n_{u} \times M}$, that enters the problem in an identical manner to $W$ in (2). In the uniformly spaced frequency case, we can transform this into the time-domain and consider,

$$
H=\mathcal{H}(g-w), \quad \text { where } g=\mathcal{F}^{-1}(G) \text { and } w=\mathcal{F}^{-1}(W) \text {, }
$$

The Hankel matrix $H$ is an affine function of the known data, $G$, and the noise $W$. We now consider $W$ (or equivalently, $w)$ to be an unknown optimization variable. For every $W$, all models consistent with the observation also must satisfy,

$$
\begin{aligned}
H & =\mathcal{H}(g-w)=\mathcal{H}(\hat{g})= \\
& {\left[\begin{array}{c}
\hat{C} \\
\hat{C} \hat{A} \\
\vdots \\
\hat{C} \hat{A}^{M-1}
\end{array}\right]\left(I-\hat{A}^{2 M}\right)^{-1}\left[\begin{array}{llll}
\hat{B} & \hat{A} \hat{B} & \cdots & \hat{A}^{M-1} \hat{B}
\end{array}\right] . }
\end{aligned}
$$

The search for the lowest order model and noise consistent with the experimental data can be expressed as,

$$
\begin{aligned}
\underset{w \in \mathcal{R}^{n_{y} \times n_{u} \times M}}{\operatorname{minimize}} & \operatorname{rank}(\mathcal{H}(g-w)) \\
\text { subject to: } & g=\mathcal{F}^{-1}(G), \text { and } w \in \mathcal{W}_{\gamma} .
\end{aligned}
$$

We have chosen to include a convex bounded (by $\gamma$ ) constraint on the noise, $w$. By appropriately defining $\mathcal{W}_{\gamma}$ we can constrain the allowable consistent noise to have additional properties. The simplest choice would be,

$$
W_{\gamma}=\left\{w \mid\|w\|_{2} \leq \gamma\right\},
$$

and this would constrain the noise in terms of its total energy over the measured frequencies. There are many other potential characterization of noise and Section III-A describes another particularly useful choice.

The advantage of the above formulation is that all solutions admit an exact state-space realization. No further approximation is required in terms of fitting the $\hat{A}, \hat{B}$ or $\hat{C}$ matrices. Section IV describes a well-known procedure for doing this step.

The disadvantage is that the rank minimization is NPhard [14] and therefore a global solution is not tractable. We therefore replace the rank objective with the increasingly popular nuclear norm objective, giving the following convex optimization problem.

Problem 1: (Uniformly spaced frequency data)

$$
\begin{aligned}
\underset{w \in \mathcal{R}^{n_{y} \times n_{u} \times M}}{\operatorname{minimize}} & \|\mathcal{H}(g-w)\|_{*} \\
\text { subject to: } & g=\mathcal{F}^{-1}(G), \text { and } w \in \mathcal{W}_{\gamma} .
\end{aligned}
$$

For a specified value of $\gamma$, the noise signal solving this problem is denoted as $\hat{w}_{\gamma}$ and the estimated impulse response coefficients are $\hat{g}_{\gamma}=g-\hat{w}_{\gamma}$. If the nuclear norm heuristic is successful then the corresponding Hankel matrix, $\mathcal{H}\left(\hat{g}_{\gamma}\right)$ is low rank and a minimal realization can be factored exactly (see Section IV).

In the non-uniformly spaced frequency sample case (Section $\mathrm{V})$ we will use an objective of the form $\|\mathcal{H}(g-w) X\|_{*}$, where $X$ is a weighting matrix. This choice does not affect the key advantages of convexity in the optimization and exact reconstruction methods via the Hankel structure.

\section{A. Spectral constraints on the noise}

One potential disadvantage of the noise constraint in (9) is that the solution to minimizing $\|\mathcal{H}(g-w)\|_{*}$ in (10) will always give an underestimate (in terms of nuclear norm) to the data. This means that the consistent noise, $w$, and the data samples, $G$ are correlated. If the user is looking for 
models compatible with the assumption that the noise arises from independent measurement noise then the result is clearly biased with respect to this assumption.

In order to have $\mathcal{W}_{\gamma}$ constrain the consistent noise to have more "noise-like" properties we use a constraint similar to that used by Paganini [23] for robust control design. A similar approach was used in [25] for an identification problem.

Define $\mathcal{W}_{\gamma, \eta}$ as the set of $M$ length real-valued sequences satisfying the constraint,

$$
\begin{aligned}
& \mathcal{W}_{\gamma, \eta}=\left\{w,\left.\left|\sum_{i=0}^{n}\right| W_{i}\right|^{2} \leq(\eta+n /(2 M-1)) \gamma^{2},\right. \\
& \text { for all } n=1, \ldots, M\} \text {, }
\end{aligned}
$$

where $W=\left\{W_{1}, \ldots, W_{M}\right\}=\mathcal{F}^{-1}(w)$. In a slight abuse of notation we will sometimes express this constraint directly in the frequency domain as $W \in \mathcal{W}_{\gamma, \eta}$ where $W=\mathcal{F}(w)$. This set is convex in the optimization variable $w$ (and $W$ ). The motivation behind choice of the constraint $w \in \mathcal{W}_{\gamma, \eta}$ is that realizations of white noise signals have cumulative spectra that are close to linear. The noise set description used by Paganini actually uses $\|w\|_{2}^{2}$ in place of $\gamma^{2}$, thereby constraining the cumulative spectrum to be linear to within a tolerance $\eta$. The definition of $\mathcal{W}_{\gamma, \eta}$ above is actually a convex relaxation of the linear cumulative spectrum constraint; it also includes smaller norm signals with less linear cumulative spectra. The relaxed constraint has been compared to a simple norm bound in [24] and found to be an effective constraint for the nuclear norm minimization based identification.

\section{B. Uniformly spaced frequency sample algorithm}

The above results are now summarized in an algorithm that explores the trade-off between model order and constrained noise size.

Algorithm 2 (Uniformly spaced frequency data):

1. Select a cumulative spectrum tolerance, $\eta>0$.

2. Calculate the impulse response matrices of the data, $g=$ $\mathcal{F}^{-1}(G)$, extending the data to negative frequencies if necessary.

3. For a series of values, $\gamma \in\left[\gamma_{\min }, \gamma_{\max }\right]$, solve Problem 1 .

4. Calculate $\operatorname{rank}\left(\mathcal{H}\left(\hat{g}_{\gamma}\right)\right)$ for each $\gamma$ and generate the trade-off plot of $\operatorname{rank}\left(\mathcal{H}\left(\hat{g}_{\gamma}\right)\right)$ versus $\gamma$.

5. For each of the values of $\operatorname{rank}\left(\mathcal{H}\left(\hat{g}_{\gamma}\right)\right)$ of interest, find the corresponding minimum $\gamma$. From $\mathcal{H}\left(\hat{g}_{\gamma}\right)$ factor the state-space estimates, $\hat{A}_{\gamma}, \hat{B}_{\gamma}$ and $\hat{C}_{\gamma}$ (see Section IV).

6. For each of the models in step 5, estimate $\hat{D}$ via leastsquares fit to the data.

This algorithm generates a series of low order models, trading off between low noise and low order. The cummulative spectrum tolerance, $\eta$, is relative with respect to $\gamma$ and typically chosen to be small. In the example in Section VI we use $\eta=0.025$.

A maximum value for $\gamma$ is easily obtained by finding the value of $\gamma$ such that $g \in \mathcal{W}_{\gamma, \eta}$. This corresponds to the noise level required to give $\hat{g}=0$. In the case of a simple norm bounded noise model this would be $\gamma_{\max }=\|g\|_{2}$. All estimated models of order greater than zero correspond to lower values of $\gamma$. These can be determined by gridding $\gamma$ or more efficiently by an adaptive or interactive search for the values of $\gamma$ where $\operatorname{rank}\left(\mathcal{H}\left(\hat{g}_{\gamma}\right)\right)$ changes.

The Hankel matrix decomposition is independent of the system feedthrough term, so if $\hat{D}$ is required a separate linear fit to the data must be performed (step 6). The above algorithm will give a series of state-space models, each corresponding to a different size for the consistent noise vector. The correct choice is a matter of model purpose and the underlying assumptions about the system.

Note that this algorithm can easily be extended to the case where a limited number of frequency data samples are missing. For each missing frequency in the vector $g-w$, insert an unconstrained optimization variable. The characterization of $W_{\gamma, \eta}$ has to be modified slightly to maintain the linear cumulative spectrum constraint. This can be useful if certain frequency data must be discarded because of significant sinusoidal noise or hum.

\section{RECONSTRUCTING STATE-SPACE ESTIMATES}

A low-rank solution to the nuclear-norm minimization problem in (10) gives a consistent noise vector, $\hat{w} \in \mathcal{R}^{n_{y} \times n_{u} \times M}$, a consistent impulse response vector, $\hat{g}=g-\hat{w}$ and an associated Hankel matrix,

$\mathcal{H}(\hat{g})=\left[\begin{array}{cccc}\hat{g}_{1} & \hat{g}_{2} & \cdots & \hat{g}_{q} \\ \hat{g}_{2} & \hat{g}_{3} & \cdots & \hat{g}_{q+1} \\ \vdots & & \ddots & \vdots \\ \hat{g}_{p} & \hat{g}_{p+1} & \cdots & \hat{g}_{p+q-1}\end{array}\right], \quad$ with $p+q-1 \leq M$.

We now wish to find, $\hat{A}, \hat{B}$ and $\hat{C}$ such that

$$
\hat{g}_{i}=\hat{C} \hat{A}^{i-1} \hat{B}, \quad i=1, \ldots, p+q-1 .
$$

In this case the $\hat{g}_{i}$ are impulse response coefficients for some underlying system. In the non-uniformly spaced data case this will be a system with the same observability matrix as the estimate and so we are only interested in obtaining $\hat{A}$ and $\hat{C}$. Note that a Hankel matrix generated by the impulse response matrices in (12) differs from the Hankel matrix given in (3) by a factor of $\left(I-A^{2 M}\right)^{-1}$. However it is straightforward to show that $A, B$ and $C$ satisfying (3) differ from $\hat{A}, \hat{B}$ and $\hat{C}$ in (12) only by a similarity transform. Either representation will suffice for fitting the frequency domain transfer function samples.

The Ho algorithm [26], [27] is one such method. To describe this algorithm we denote the time shifted variable by,

$$
z^{-1} \hat{g}=\left[\begin{array}{llll}
\hat{g}_{2}^{T} & \hat{g}_{3}^{T} & \cdots & \hat{g}_{M+1}^{T}
\end{array}\right]^{T},
$$

and an associated Hankel matrix, $\mathcal{H}\left(z^{-1} \hat{g}\right)$, which now requires $p+q \leq M$ in order to be constructed from the available data.

The Ho algorithm can be expressed as follows:

1. Find matrices, $Q$ and $P$ such that,

$$
Q \mathcal{H}(\hat{g}) P=\left[\begin{array}{l}
Q_{1} \\
Q_{2}
\end{array}\right] \mathcal{H}(\hat{g})\left[\begin{array}{ll}
P_{1} & P_{2}
\end{array}\right]=\left[\begin{array}{cc}
I_{n_{x}} & 0 \\
0 & 0
\end{array}\right],
$$


where $n_{x}=\operatorname{rank}(\mathcal{H}(\hat{g}))$. The $Q$ and $P$ matrix partitions are commensurate with the identity matrix, $I_{n_{x}}$. Note that $Q$ and $P$ are easily calculated from a singular value decomposition of $\mathcal{H}(\hat{g})$.

2. Form the realization:

$$
\begin{aligned}
\hat{A} & =Q_{1} \mathcal{H}\left(z^{-1} \hat{g}\right) P_{1}, \\
\hat{B} & =Q_{1} \mathcal{H}(\hat{g})\left[\begin{array}{c}
I_{n_{u}} \\
0
\end{array}\right], \\
\hat{C} & =\left[\begin{array}{ll}
I_{n_{y}} & 0
\end{array}\right] \mathcal{H}(\hat{g}) P_{1} .
\end{aligned}
$$

The resulting realization is of order $n_{x}$ and is minimal (with respect to all realizations matching the impulse response coefficients required to form $\mathcal{H}(\hat{g})$ and $\left.\mathcal{H}\left(z^{-1} \hat{g}\right)\right)$.

Underlying this algorithm is the assumption that $\operatorname{rank}(\mathcal{H}(\hat{g}))=\operatorname{rank}\left(\mathcal{H}\left(z^{-1} \hat{g}\right)\right)$. If this is not satisfied there is a construction (given in [28]) to extend $\hat{g}$ with additional elements such that the rank condition is satisfied for larger Hankel matrices constructed from the extended $\hat{g}$ vector. This allows the algorithm to proceed and return a realization. Note however that the order of the minimal realization, $n_{x}$, will in this case be equal to $M$.

The rank condition on the shifted Hankel matrix is actually necessary. To see this note that if, for example,

$$
\mathcal{H}\left(z^{-1} \hat{g}\right)=\left[\begin{array}{c}
0 \\
\vdots \\
0 \\
1
\end{array}\right]\left[\begin{array}{llll}
0 & \cdots & 0 & 1
\end{array}\right] \in \mathcal{R}^{p \times q},
$$

then $\operatorname{rank}\left(\mathcal{H}\left(z^{-1} \hat{g}\right)\right)=1$ but $\operatorname{rank}(\mathcal{H}(\hat{g}))=0$ and the minimal realization is $\hat{g}(z)=z^{-p-q+1}$ which is clearly of high order. Interestingly this pathology also applies to the standard subspace identification methods. It is clearly not a practical issue in that case and we conjecture that it is not a practical issue for nuclear norm minimization approaches either.

\section{NON-UNIFORMLY SPACED FREQUENCY SAMPLES}

In the general case, we are given $M$ noisy frequency domain estimates, $G_{n}, n=1, \ldots, M$, measured at distinct irregularly spaced frequencies, $\mathrm{e}^{j \omega_{1}}, \ldots, \mathrm{e}^{j \omega_{M}}$. As in the previous case we are looking for noise vectors, $W$, and low-order models, $\hat{G}\left(\mathrm{e}^{j \omega_{n}}\right)$, consistent with the measured data-in otherwords, satisfying (2).

The basic formulation of the problem follows that given in [7]. In the frequency domain, the noise-free state-space equations of the system $G\left(\mathrm{e}^{j \omega}\right)$ are,

$$
\begin{aligned}
\mathrm{e}^{j \omega} X(\omega) & =A X(\omega)+B U(\omega), \\
Y(\omega) & =C X(\omega)+D U(\omega),
\end{aligned}
$$

where $Y(\omega), X(\omega)$ and $U(\omega)$ are the frequency domain representations of the output, state and input signals respectively. Consider applying a sinusoid of frequency $\omega_{n}$ to input $j$, giving, in the frequency-domain, $U\left(\omega_{n}\right)=e_{j}$. Denote the resulting state solution by $X^{j}\left(\omega_{n}\right)$. If this procedure is repeated for all inputs, and the resulting state solutions are stacked column-wise,

$$
X^{C}\left(\omega_{n}\right)=\left[X^{1}\left(\omega_{n}\right) X^{2}\left(\omega_{n}\right) \cdots X^{n_{u}}\left(\omega_{n}\right)\right] \in \mathcal{C}^{n_{x} \times n_{u}},
$$

then the transfer function, at frequency $\omega_{n}$, can be written as,

$$
\begin{aligned}
\mathrm{e}^{j \omega_{n}} X^{C}\left(\omega_{n}\right) & =A X^{C}\left(\omega_{n}\right)+B \\
G\left(\mathrm{e}^{j \omega_{n}}\right) & =C X^{C}\left(\omega_{n}\right)+D
\end{aligned}
$$

By recursively multiplying the second equation by $\mathrm{e}^{j \omega_{n}}(p-1$ times) and substituting in the first, we get,

$$
\begin{array}{r}
{\left[\begin{array}{c}
1 \\
\mathrm{e}^{j \omega_{n}} \\
\vdots \\
\mathrm{e}^{j(p-1) \omega_{n}}
\end{array}\right] \otimes G\left(\mathrm{e}^{j \omega_{n}}\right)} \\
=\mathcal{O} X^{C}\left(\mathrm{e}^{j \omega_{n}}\right)+\Gamma\left[\begin{array}{c}
1 \\
\mathrm{e}^{j \omega_{n}} \\
\vdots \\
\mathrm{e}^{j(p-1) \omega_{n}}
\end{array}\right],
\end{array}
$$

where $\mathcal{O}$ is the extended observability matrix and

$$
\Gamma=\left[\begin{array}{cccc}
D & 0 & \cdots & 0 \\
C B & D & \cdots & 0 \\
\vdots & \vdots & & \vdots \\
C A^{p-2} B & C A^{p-1} B & \cdots & D
\end{array}\right] .
$$

Now apply (16) at frequencies, $\omega_{n}, n=1, \cdots, M$, and stack the equations columnwise to get,

$$
\begin{aligned}
& \left(V \otimes I_{n_{y}}\right) \operatorname{diag}\left(G\left(\mathrm{e}^{j \omega_{1}}\right), \cdots, G\left(\mathrm{e}^{j \omega_{M}}\right)\right) \\
& =\mathcal{O}\left[\begin{array}{lll}
X^{C}\left(\omega_{1}\right) & \cdots & X^{C}\left(\omega_{M}\right)
\end{array}\right]+\Gamma\left(V \otimes I_{n_{u}}\right),
\end{aligned}
$$

where $V$ is the Vandermonde matrix given by,

$$
V=\left[\begin{array}{cccc}
1 & 1 & \cdots & 1 \\
\mathrm{e}^{j \omega_{1}} & \mathrm{e}^{j \omega_{2}} & \cdots & \mathrm{e}^{j \omega_{M}} \\
\mathrm{e}^{j 2 \omega_{1}} & \mathrm{e}^{j 2 \omega_{2}} & \cdots & \mathrm{e}^{j 2 \omega_{M}} \\
\vdots & \vdots & & \vdots \\
\mathrm{e}^{j(p-1) \omega_{1}} & \mathrm{e}^{j(p-1) \omega_{2}} & \cdots & \mathrm{e}^{j(p-1) \omega_{M}}
\end{array}\right]
$$

For simplicity we will denote block-Vandermonde matrices by $V_{n_{y}}=\left(V \otimes I_{n_{y}}\right)$ and similarly for $V_{n_{u}}$. Now consider the case where we have chosen the number of (block) rows, $p$, to be more than the assumed order of the underlying system. Furthermore, assume that we have also measured $M>p$ frequencies. Under these assumptions $V_{n_{u}}$ has a right nullspace which we denote by $V_{n_{u}}^{\perp}$. We will further simplify the notation by defining,

$$
\mathcal{X}=\left[\begin{array}{lll}
X^{C}\left(\omega_{1}\right) & \cdots & X^{C}\left(\omega_{M}\right)
\end{array}\right]
$$

Multiplying (17) on the right by $V_{n_{u}}^{\perp}$ gives,

$$
V_{n_{y}} \operatorname{diag}(G) V_{n_{u}}^{\perp}=\mathcal{O} \mathcal{X} V_{n_{u}}^{\perp} .
$$

In the noisy data case the true system can be expressed in terms of the measured data $G$, and noise $W$, as,

$$
\left.V_{n_{y}} \operatorname{diag}(G-W)\right) V_{n_{u}}^{\perp}=\mathcal{O} \mathcal{X} V_{n_{u}}^{\perp} .
$$


This relationship is used to formulate the search for candidate noise and low-order model combinations consistent with the observed data. This motivates us to consider the following optimization problem as the basis for our algorithm.

Problem 3 (Non-uniformly spaced frequency data): Given frequency domain estimates, $G \in \mathcal{C}^{n_{y} \times n_{u} \times M}$ at distinct $M$ frequencies, $\mathrm{e}^{j \omega_{n}}, n=1, \ldots, M$; create $V_{n_{y}}$ and from any full-rank $V^{\perp}$ create $V_{n_{u}}^{\perp}$.

Solve:

$$
\begin{aligned}
\underset{W \in \mathcal{C}^{n_{y} \times n_{u} \times M}}{\operatorname{minimize}} & \left\|V_{n_{y}} \operatorname{diag}(G-W) V_{n_{u}}^{\perp}\right\|_{*} \\
\text { subject to: } & W \in \mathcal{W}_{\gamma} \quad\left(\text { or } W \in \mathcal{W}_{\gamma, \eta}\right) .
\end{aligned}
$$

We will show that this yields a low order approximation to the data by showing that $V_{n_{y}} \operatorname{diag}(G-W) V_{n_{u}}^{\perp}$ has the range space, rank and shift structure of a Hankel matrix corresponding to a low-order realization. From this Hankel matrix the $\hat{A}$ and $\hat{C}$ matrices of the realization can be exactly calculated (via (13) and (15)).

Lemma 4: Given a full-rank, block-Vandermonde matrix, $V_{n_{y}}$, create $V_{n_{u}}^{\perp}$ from any full rank matrix, $V^{\perp}$ such that $V V^{\perp}=0$. Then for all $W \in \mathcal{W}_{\gamma} \subset \mathcal{C}^{n_{y} \times n_{u} \times M}$ there exists a factorization,

$$
V_{n_{y}} \operatorname{diag}(G-W) V_{n_{u}}^{\perp}=H X
$$

where $H \in \mathcal{C}^{n_{y} p \times n_{u}(M-p)}$ is a block-Hankel matrix (with blocks of size $n_{y} \times n_{u}$ ) having shift structure, and $X \in$ $\mathcal{C}^{n_{u}(M-p) \times n_{u}(M-p)}$ is full rank.

As $X$ is full rank, it follows that,

$$
\operatorname{rank}\left(V_{n_{y}} \operatorname{diag}(G-W) V_{n_{u}}^{\perp}\right)=\operatorname{rank}(H),
$$

and so a low-rank solution to the nuclear-norm minimization gives a low-rank Hankel matrix, $H$, with an extended observability space matching that of $\hat{G}\left(\mathrm{e}^{j \omega}\right)$ in (2). From $H$ we obtain, using the approach in Section IV, output and state matrices, $\hat{C}$ and $\hat{A}$ generating this extended observability space.

Unlike the uniformly spaced frequency case, the formulation of $H$ does not necessarily lead to a meaningful interpretation of the input matrix, $\hat{B}$, given by (14). However given $\hat{C}$ and $\hat{A}$, one can create a linear optimization problem (as is done in [7]) to give $\hat{B}$ and $\hat{D}$ (if required).

The proof of Lemma 4 depends on the construction of a particular $V^{\perp}$ which-as it is of potential independent interest-we give in Lemma 5 below. For simplicity this is stated in the SISO case.

Lemma 5: Given a Vandermonde matrix, $V \in \mathcal{C}^{p \times M}$, with $p<M$, generated by a vector $v \in \mathcal{C}^{M}$ such that $v_{i} \neq v_{j}$ if $i \neq j$, and a vector, $x \in \mathcal{C}^{M}$, there exists a full-rank matrix $\hat{V}^{\perp} \in \mathcal{C}^{M \times M-p}$, such that,

$$
V \hat{V}^{\perp}=0,
$$

and $V \operatorname{diag}(x) \hat{V}^{\perp}$ has Hankel and shift structure for all $x \in$ $\mathcal{C}^{M}$.
Proof of Lemma 5: The Vandermonde matrix, $V \in$ $\mathcal{C}^{p \times M}$, generated by $v \in \mathcal{C}^{M}$, can be written as,

$$
\begin{gathered}
V=\left[\begin{array}{cccc}
1 & 1 & \cdots & 1 \\
v_{1} & v_{2} & \cdots & v_{M} \\
v_{1}^{2} & v_{2}^{2} & \cdots & v_{M}^{2} \\
\vdots & \vdots & & \vdots \\
v_{1}^{p-1} & v_{2}^{p-1} & \cdots & v_{M}^{p-1}
\end{array}\right]=\left[\begin{array}{c}
1_{M}^{T} \\
1_{M}^{T} \operatorname{diag}(v) \\
\vdots \\
1_{M}^{T}(\operatorname{diag}(v))^{p-1}
\end{array}\right] \\
=\left[1_{M}^{T}(\operatorname{diag}(v))^{i-1}\right]_{i=1, \ldots, p}
\end{gathered}
$$

where $1_{M} \in \mathcal{R}^{M}$ denotes the vector with all elements equal to one. Now augment $V$ with $M-p$ additional rows, generated in the obvious manner,

$$
V_{M}:=\left[1_{M}^{T}(\operatorname{diag}(v))^{i-1}\right]_{i=1, \ldots, M} \in \mathcal{C}^{M \times M} .
$$

As the elements of $v$ are distinct both $V$ and $V_{M}$ are full-rank. By taking a QR decomposition of $V_{M}^{*}$ we can find a unitary $Q_{M}$ and lower triangular $R_{M}$ such that $V_{M} Q_{M}=R_{M}$. As $V_{M}$ is full rank $R_{M}$ has no zero elements on the main diagonal. This decomposition can be used to form the lower triangular matrix,

$$
V_{M} Q_{M}=\left[1_{M}^{T} Q_{M}\left(Q_{M}^{*} \operatorname{diag}(v) Q_{M}\right)^{i-1}\right]_{i=1, \ldots, M} .
$$

Now define $A=Q_{M}^{*} \operatorname{diag}(v) Q_{M}$ and note that $1_{M}^{T} Q_{M}=$ $\alpha e_{1}^{T}$ for some $\alpha \in \mathcal{C}$. Because $V_{M} Q_{M}$ is lower triangular it is possible to show that all of the non-zero elements of $A$ are on the first superdiagonal and below. Because $R_{M}$ has no zero diagonal elements, $A$ has no zero elements on the first superdiagonal.

Now divide $\mathcal{C}^{M}$ into two subspaces by partitioning as,

$$
\mathcal{C}^{M}=\left[\begin{array}{c}
\mathcal{X}_{1} \\
0
\end{array}\right] \bigcup\left[\begin{array}{c}
0 \\
\mathcal{X}_{2}
\end{array}\right], \quad \text { with } \mathcal{X}_{1} \in \mathcal{C}^{p} \text { and } \mathcal{X}_{2} \in \mathcal{C}^{M-p} \text {. }
$$

Note that for all $x_{2} \in \mathcal{X}_{2}$, the zero structure of $A$ implies that,

$$
\alpha e_{1}^{T} A^{i-1}\left[\begin{array}{c}
0 \\
x_{2}
\end{array}\right]=0 \quad \text { for } i=0,1, \ldots, p-1 .
$$

An identical argument shows that,

$$
A^{j-1} e_{M} \in\left[\begin{array}{c}
0 \\
\mathcal{X}_{2}
\end{array}\right] \quad \text { for all } j=0,1, \ldots, M-p .
$$

To complete the construction define

$$
\hat{V}^{\perp}:=Q_{M}\left[A^{j-1} e_{M}\right]_{j=1, \ldots, M-p},
$$

and observe that,

$$
\begin{aligned}
V \hat{V}^{\perp} & =\left[1_{M}^{T}(\operatorname{diag}(v))^{i-1}\right]_{i=1, \ldots, p} Q_{M}\left[A^{j-1} e_{M}\right]_{j=1, \ldots, M-p} \\
& =\left[\alpha e_{1}^{T} A^{i-1}\right]_{i=1, \ldots, p}\left[A^{j-1} e_{M}\right]_{j=1, \ldots, M-p}, \\
& =0,
\end{aligned}
$$


as, by (21), the right-hand factor is an element of $\left[\begin{array}{ll}0^{T} & \mathcal{X}_{2}^{T}\end{array}\right]^{T}$, and, as shown in (20), multiplying the left-hand factor by any such element gives zero. As $A$ has non-zero elements on the first superdiagonal each vector,

$$
v_{j}^{\perp}=A^{j-1} e_{M}, \quad j=1, \ldots, M-p,
$$

has exactly one more non-zero component than the prior vector, ensuring that all vectors thus generated are linearly independent. Therefore $\hat{V}^{\perp}$, defined in (22), is full-rank.

To include $x$ and construct the Hankel matrix note that,

$$
\begin{aligned}
V & \operatorname{diag}(x) \hat{V}^{\perp} \\
= & {\left[1_{M}^{T}(\operatorname{diag}(v))^{i-1}\right]_{i=1, \ldots, p} \operatorname{diag}(x) \hat{V}^{\perp} } \\
= & {\left[1_{M}^{T} \operatorname{diag}(x)(\operatorname{diag}(v))^{i-1}\right]_{i=1, \ldots, p} \hat{V}^{\perp} } \\
= & {\left[x^{T}(\operatorname{diag}(v))^{i-1}\right]_{i=1, \ldots, p} \hat{V}^{\perp} } \\
= & {\left[x^{T}(\operatorname{diag}(v))^{i-1}\right]_{i=1, \ldots, p} Q_{M}\left[A^{j-1} e_{M}\right]_{j=1, \ldots, M-p} } \\
= & {\left[x^{T} Q_{M} A^{i-1}\right]_{i=1, \ldots, p}\left[A^{j-1} e_{M}\right]_{j=1, \ldots, M-p} } \\
= & {\left[x^{T} Q_{M} A^{i+j-2} e_{M}\right]_{i=1, \ldots, p}{ }_{j=1, \ldots, M-p} }
\end{aligned}
$$

which clearly has Hankel structure. It also has shift structure where $x^{T} Q_{M}$ plays the role of the $C$ matrix.

It is easily verified that $V \operatorname{diag}(x) \hat{V}^{\perp}$ is invariant to the addition of a constant to all components of $x$;

$$
\begin{aligned}
V \operatorname{diag}\left(x+\beta 1_{M}\right) \hat{V}^{\perp} & =V \operatorname{diag}(x) \hat{V}^{\perp}+\beta V \hat{V}^{\perp} \\
& =V \operatorname{diag}(x) \hat{V}^{\perp}, \quad \text { for all } \beta \in \mathcal{C} .
\end{aligned}
$$

In the identification context this means that $V \operatorname{diag}(G-W) \hat{V}^{\perp}$ is independent of the state-space $\hat{D}$ term.

Proof of Lemma 4:: First note that the construction given for $V$ in Lemma 5 applies to the block-Vandermonde case with only minor modifications. This can be shown by constructing, $\hat{V}_{n_{u}}^{\perp}=\hat{V}^{\perp} \otimes I_{n_{u}}$, and noting that,

$V_{n_{u}} \hat{V}_{n_{u}}^{\perp}=\left(V \otimes I_{n_{u}}\right)\left(\hat{V}^{\perp} \otimes I_{n_{u}}\right)=\left(V \hat{V}^{\perp}\right) \otimes\left(I_{n_{u}} I_{n_{u}}\right)=0$.

A similar commuting argument applies where needed in the expression of $V \operatorname{diag}(x) \hat{V}^{\perp}$ in the proof of Lemma 5 above.

As $\hat{V}^{\perp}$ is full rank, all $V^{\perp}$ such that $V V^{\perp}=0$, can be expressed as,

$$
V^{\perp}=\hat{V}^{\perp} X, \text { for some } X \in \mathcal{C}^{(M-p) \times(M-p)} .
$$

If $V^{\perp}$ is full rank, the $X$ must also be full rank. This, together with Lemma 5, immediately gives the factorization required in (19).

\section{A. An alternative real-valued formulation}

The formulation in Problem 3 and Lemma 4 used a complex-valued formulation, whereas in practical identification problems we are usually more interested in finding a realvalued representation. To formulate a real-valued problem we define a lifting, $\mathcal{L}(X): \mathcal{C}^{r \times c} \longrightarrow \mathcal{R}^{2 r \times 2 c}$, via,

$$
\mathcal{L}(X)=\left[\begin{array}{cc}
\operatorname{real}(X) & \operatorname{imag}(X) \\
-\operatorname{imag}(X) & \operatorname{real}(X)
\end{array}\right] .
$$

For notational simplicity we further define,

$$
\mathcal{L}_{\text {ri }}(X)=[\operatorname{real}(X) \operatorname{imag}(X)] .
$$

We now follow a commonly used approach (as is done in [7]) by noting that the extended observability matrix is real-valued and so (18) is instead expressed as,

$$
\mathcal{L}_{\text {ri }}\left(V_{n_{y}} \operatorname{diag}(G-W) V_{n_{u}}^{\perp}\right)=\mathcal{O} \mathcal{L}_{\text {ri }}\left(\mathcal{X} V_{n_{u}}^{\perp}\right) .
$$

We can now state a real-valued version of Lemma 4.

Lemma 6: Given a full-rank, block-Vandermonde matrix, $V_{n_{y}}$, and any full rank matrix, $V_{n_{u}}^{\perp}$ such that $V V^{\perp}=0$, then for all $W_{n} \in \mathcal{W}_{\gamma} \subset \mathcal{C}^{n_{y} \times n_{u}}$ there exists a factorization,

$$
\mathcal{L}_{\text {ri }}\left(V_{n_{y}} \operatorname{diag}(G-W) V_{n_{u}}^{\perp}\right)=H X,
$$

where $H \in \mathcal{R}^{n_{y} p \times n_{u}(2 M-p)}$ is a block-Hankel matrix (with blocks of size $n_{y} \times n_{u}$ ) having shift structure, and $X \in$ $\mathcal{R}^{n_{u}(2 M-p) \times n_{u}(2 M-p)}$ is full rank.

We again have an affine relationship between $H$ and variables that will subsequently be used as optimization variables, $\operatorname{real}\left(W_{n}\right)$ and $\operatorname{imag}\left(W_{n}\right)$. The rank relationship between $H$ and the left-hand side of (25) is also preserved.

Proof of Lemma 6: This follows the same argument as the proof of Lemmas 4, and 5 using variables defined by the lifting in (24), This lifting is linear and so preserves all of the matrix algebra we require in the proof. Now we can express $\mathcal{L}_{\text {ri }}(V)$ as,

$$
\mathcal{L}_{\mathrm{ri}}(V)=\left[\mathcal{L}_{\mathrm{ri}}(\operatorname{diag}(v))^{i-1}\right]_{i=1, \ldots, p} \in \mathcal{R}^{p \times 2 M},
$$

and note that,

$$
\begin{aligned}
\mathcal{L}_{\mathrm{ri}}(V \operatorname{diag}(x))=\left[\mathcal{L}_{\mathrm{ri}}(\operatorname{diag}(v))^{i-1}\right]_{i=1, \ldots, p} \mathcal{L}(\operatorname{diag}(x)) \\
=\left[\mathcal{L}_{\mathrm{ri}}\left(x^{T}\right) \mathcal{L}(\operatorname{diag}(v))^{i-1}\right]_{i=1, \ldots, p},
\end{aligned}
$$

as $\mathcal{L}(\operatorname{diag}(v))$ and $\mathcal{L}(\operatorname{diag}(x))$ commute. Using the same line of argument as in Lemma 5, one can add an addition $2 M-p$ rows to the matrix giving,

$$
\mathcal{L}_{\text {ri }}\left(V_{2 M}\right)=\left[\mathcal{L}_{\text {ri }}(\operatorname{diag}(v))^{i-1}\right]_{i=1, \ldots, 2 M} \in \mathcal{R}^{2 M \times 2 M} .
$$

Again find the unitary matrix, $Q_{2 M} \in \mathcal{R}^{2 M \times 2 M}$, which will multiply the above to give a lower triangular result. An identical construction is employed to find the required parametrization of the null-space,

$$
\mathcal{L}_{\text {ri }}(V)^{\perp} \in \mathcal{R}^{2 M \times(2 M-p)} .
$$


The construction of the Hankel matrix uses identical algebra to that in Lemma 5. The remainder of the proof uses the same multivariable argument as Lemma 4.

For a noise set with size specified by $\gamma$, this leads to the following statement of the optimization problem.

Problem 7 (Real-valued realization): Given frequency domain estimates, $G \in \mathcal{C}^{n_{y} \times n_{u} \times M}$ at distinct $M$ frequencies, $\mathrm{e}^{j \omega_{n}}, n=1, \ldots, M$; create $V_{n_{y}}$ and from any full-rank $V^{\perp}$ create $V_{n_{u}}^{\perp}$.

Solve:

$$
\begin{aligned}
\underset{W \in \mathcal{C}^{n_{y} \times n_{u} \times M}}{\operatorname{minimize}} & \left\|\mathcal{L}_{\mathrm{ri}}\left(V_{n_{y}} \operatorname{diag}(G-W) V_{n_{u}}^{\perp}\right)\right\|_{*} \\
\text { subject to: } & W \in \mathcal{W}_{\gamma, \eta} .
\end{aligned}
$$

The minimizing solution is denoted by $\hat{W}_{\gamma}$. The associated Hankel matrix (as given in (25)) is $\mathcal{H}\left(\hat{g}_{\gamma}\right)$.

\section{B. Non-uniformly spaced frequency algorithm}

To summarize the above results, and show their application to the identification problem, we present an algorithm for the general non-uniformly spaced frequency case.

Algorithm 8 (Non-uniformly spaced frequency data):

1. Select a cumulative spectrum tolerance, $\eta>0$.

2. Calculate the Vandermonde matrix $V$ and $\hat{V}^{\perp}$. Apply an optional weighting $X$, to give $V^{\perp}=\hat{V}^{\perp} X$. From these form $V_{n_{y}}$, and $V_{n_{u}}^{\perp}$.

3. For a series of values, $\gamma \in\left[\gamma_{\min }, \gamma_{\max }\right]$, solve Problem 7 .

4. Calculate $\operatorname{rank}\left(\mathcal{H}\left(\hat{g}_{\gamma}\right)\right)$ for each $\gamma$ and generate the trade-off plot of $\operatorname{rank}\left(\mathcal{H}\left(\hat{g}_{\gamma}\right)\right)$ versus $\gamma$.

5. For each of the values of $\operatorname{rank}\left(\mathcal{H}\left(\hat{g}_{\gamma}\right)\right)$ of interest, find the corresponding minimum $\gamma$. From $\mathcal{H}\left(\hat{g}_{\gamma}\right)$ factor the state-space estimates, $\hat{A}_{\gamma}$ and $\hat{C}_{\gamma}$ (see Section IV).

6. For each of the models in step 5 , estimate $\hat{B}$ and $\hat{D}$ by a linear fit to $G-\hat{W}_{\gamma}$.

The weighting matrix, $X$, affects only the numerical properties of the optimization in Problem 7 and a suitable initial default is $X=I$. We have found that the convergence properties are often improved by choosing $X$ so that $V_{n_{u}}^{\perp}$ has a low condition number.

\section{NUMERICAL EXAMPLE}

An irregularly spaced frequency example is studied to illustrate the use of the nuclear norm minimization methods given above. The data and noise are simulated which allows us to examine the properties of the "true" system, denoted by $G_{\text {true }}$. We have chosen $G_{\text {true }}$ as a stable 6th order system, normalized to have a maximum magnitude gain of unity.

Measurement data was simulated at $M=40$ irregularly spaced points around the positive frequency arc of the unit circle. The simulated measurement data was corrupted by zero mean, normally distributed noise with a variance of $\lambda=0.002$. This is a very high noise level compared to the less observable/controllable modes of the system and under these circumstances we should not expect to be able to recover much detail about the system.

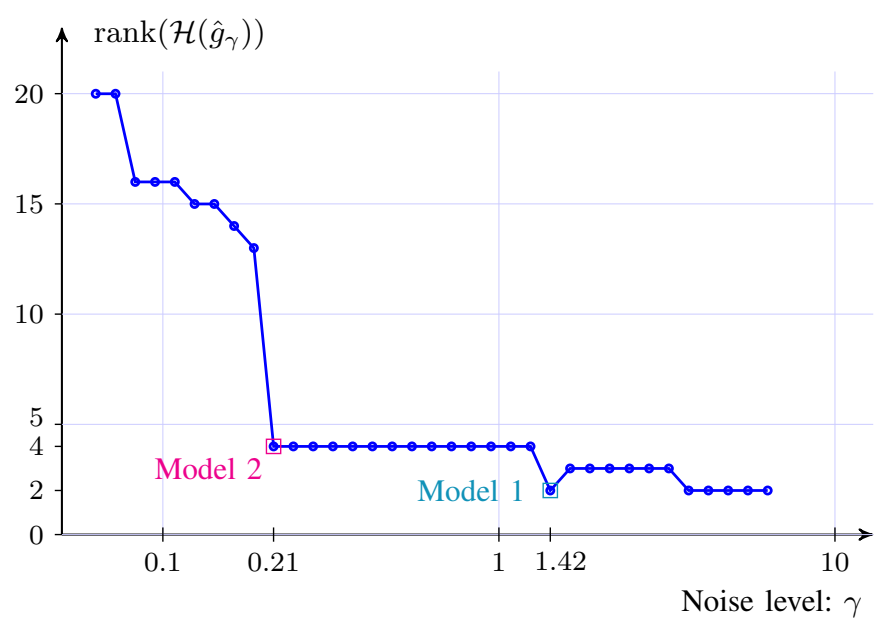

Fig. 1. Nuclear norm minimization result: $\operatorname{rank}\left(\mathcal{H}\left(\hat{g}_{\gamma}\right)\right)$ as a function of $\gamma$. The $\operatorname{rank}\left(\mathcal{H}\left(\hat{g}_{\gamma}\right)\right)$ versus $\gamma$ trade-off points for the two selected low order models are identified.

The steps outlined in Algorithm 8 were performed in order to estimate two low order models for the system. The cumulative spectrum linearity tolerance was chosen as $\eta=0.025$. A logarithmically spaced vector of $\gamma$ values was chosen for study. This covered two orders of magnitude between 0.063 and 6.30. The maximum value of $\gamma$ is close to the 2-norm of the raw data vector. This heuristic is easily adjusted depending on the rank of $\mathcal{H}\left(\hat{g}_{\gamma}\right)$ found for the maximum value of $\gamma$. The nuclear norm minimization weight $X$ was chosen such that $V^{\perp}$ was an orthonormal basis for the null space.

The optimization problem in Algorithm 8 was solved using the MATLAB CVX toolbox [29], [30]. The MATLAB code and data for preproducing the example is available for download from the author's website.

Figure 1 shows the rank of the solutions, $\mathcal{H}\left(\hat{g}_{\gamma}\right)$. Note that for all $\gamma>0.2$ low rank solutions are found showing that the nuclear norm heuristic performs very well. The rank of $\mathcal{H}\left(\hat{g}_{\gamma}\right)$ grows as $\gamma$ decreases below $\gamma=0.2$. In these cases the frequency domain data fit, $\hat{G}+\hat{W}_{\gamma}$, is fitting the noise response. Of course we can only make this statement when we know the underlying system response. This observation has been found to hold in a large number of randomly generated simulations.

Although $\left\|\mathcal{H}\left(\hat{g}_{\gamma}\right)\right\|_{*}$ will be monotonically non-increasing, there is no theoretical guarantee that this will hold for $\operatorname{rank}\left(\mathcal{H}\left(\hat{g}_{\gamma}\right)\right)$. In this example, the $\operatorname{rank}\left(\mathcal{H}\left(\hat{g}_{\gamma}\right)\right)$ curve is not monotonically decreasing with $\gamma$, although for the majority of examples studied this is the case. Here the rank 3 models have one singular value significantly smaller than the others. Their responses are predominantly second order.

On the basis of Figure 1, two models are selected for realization and further analysis. These are the 2nd and 4th order models labeled and one can see that they correspond to the smallest noise giving the specified rank. Figure 2 gives a more detailed analysis of the singular values corresponding to each case. The most striking feature illustrated is that the ranks of $\mathcal{H}\left(\hat{g}_{\gamma}\right)$ for the two models chosen are unambiguously determined. There is step in singular values of at least 10 


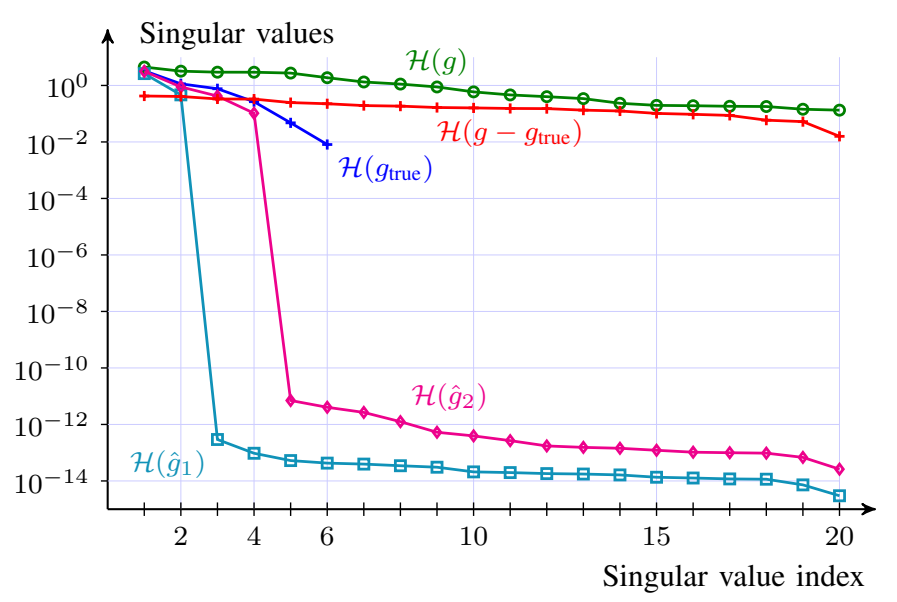

Fig. 2. Comparison between the singular values of the Hankel matrices formed from the data, $\mathcal{H}(g)$, and the two low order models, $\mathcal{H}\left(\hat{g}_{1}\right)$ and $\mathcal{H}\left(\hat{g}_{2}\right)$, corresponding to models 1 and 2, identified in Figure 1. Also shown are the singular values of the Hankel matrices generated by the true system, $\mathcal{H}\left(g_{\text {true }}\right)$, and by the noise alone, $\mathcal{H}\left(g-g_{\text {true }}\right)$.

orders of magnitude. This further supports the use of the nuclear norm as a rank minimization heuristic. The singular values of the Hankel matrix associated with the data, $\mathcal{H}(g)$, are also shown. These are the singular values that one would have to analyze in the standard subspace identification method. There is no clear basis for rank selection in this case-all of the singular values lie within a two-order of magnitude range. The singular values due to noise alone are shown and the reason for the difficulties with standard subspace identification are obvious; the size of noise contribution is close to the singular values of the true system alone, $\mathcal{H}\left(g_{\text {true }}\right)$, and is even larger than the smallest three system singular values.

The two low order models were realized using the Ho algorithm in Section IV. Figure 3 shows the magnitude and phase responses of the low order models and compare these to both the data and the system $G_{\text {true }}$. The 4th order model is clearly better and shows some of the oscillatory mode response around $\omega=0.6$ radians/second. Comparison with the true system shows that the 4th order model underestimates this mode. Given the very high noise level, this can be considered a reasonably good result.

\section{CONCLUSIONS}

We have developed nuclear norm based minimization methods for frequency domain subspace identification, in both the uniform and non-uniform frequency sample cases. One of the key aspects of this work is the parametrization of the model in a way that gives an exact realization. This avoids the need to estimate the state-space matrices associated with the extended observability space; they can be determined exactly by numerically stable factorizations. We expect that this parametrization will also be useful in the development of efficient optimization algorithms for this problem and this aspect is the focus of on-going work.

Another advantage of the parametrizations given here is that they are linear functions of the noise. This means that a wide variety of noise constraints can be applied without

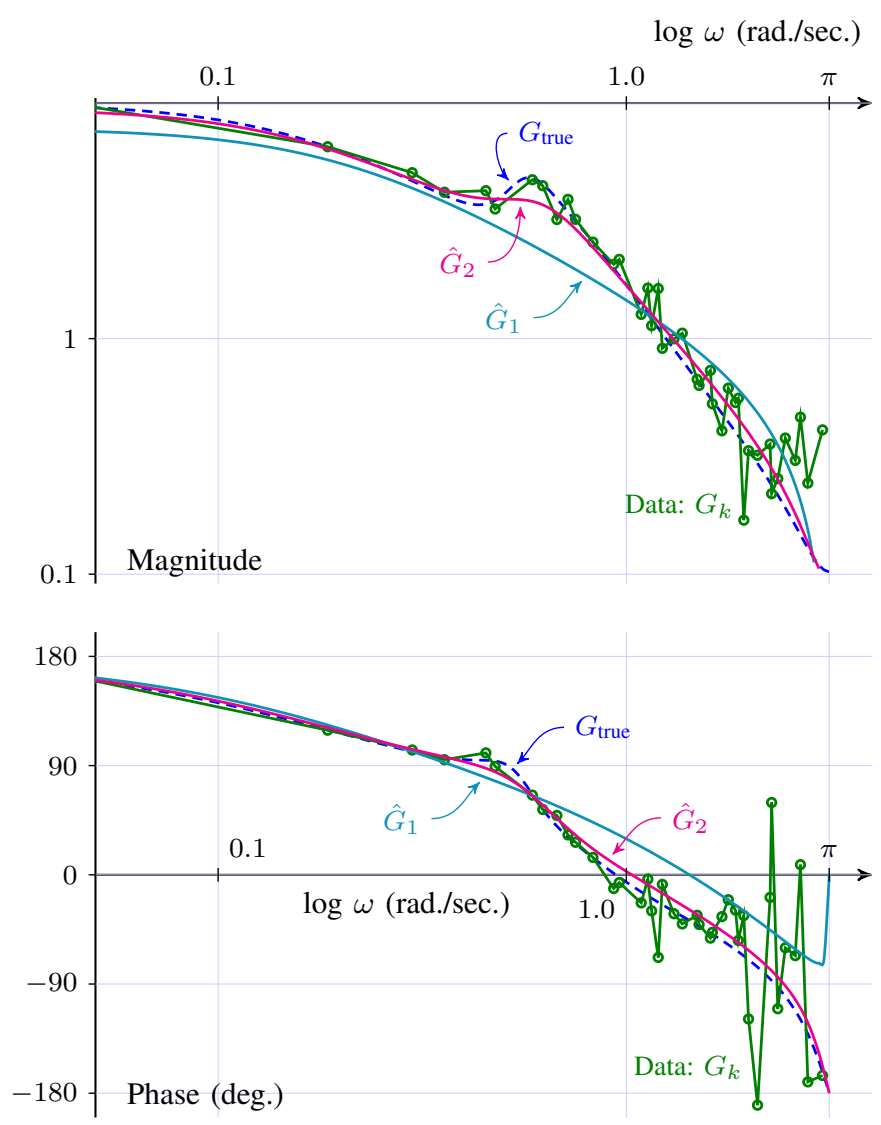

Fig. 3. Comparison of the frequency responses of the low order models, $\hat{G}_{1}$ and $\hat{G}_{2}$, and the observed data, $G_{k}$, with data points indicated by circles. Also shown is the frequency response of the true system, $G_{\text {true }}$ (dashed line).

requiring modification of the minimization objective function. This avoids the problem of nonlinear noise influence that arises in standard subspace identification methods. The cumulative spectral constraint presented here has been found to be useful and is less prone to underestimation than a standard norm constraint.

The focus of this work has been frequency domain estimation. However the standard formulation of time-domain subspace identification problems uses a matrix structure similar to the one developed in Section V. We expect that the work described here can also be applied in the time-domain case and this is also a topic of ongoing research.

\section{ACKNOWLEDGEMENTS}

The author would like to thank Tomas McKelvey, Stephen Boyd, Håkan Hjalmarsson, Bo Wahlberg and Ben Rechts for useful discussions on this topic.

\section{REFERENCES}

[1] M. Viberg, B. Ottersten, B. Wahlberg, and L. Ljung, "A statistical perspective on state-space modeling using subspace methods," in Proc. IEEE Control and Decision Conf., pp. 1337-1342, 1991.

[2] M. Verhaegen and P. Dewilde, "Subspace model identification Part 1. The output-error state-space model identification class of algorithms," Int. J. Control, vol. 56, pp. 1187-1210, Nov. 1992.

[3] M. Verhaegen and P. Dewilde, "Subspace model identification Part 2. Analysis of the elementary output-error state-space model identification algorithm," Int. J. Control, vol. 56, pp. 1211-1241, Nov. 1992. 
[4] M. Verhaegen, "Subspace model identification Part 3. Analysis of the ordinary output-error state-space model identification algorithm," Int. J. Control, vol. 58, pp. 555-586, Sept. 1993.

[5] P. Van Overschee and B. De Moor, "N4SID: subspace algorithms for the identification of combined deterministic-stochastic systems," Automatica, vol. 30, no. 1, pp. 75-93, 1994.

[6] B. De Schutter, "Minimal state-space realization in linear system theory: an overview," J. Computational and Applied Mathematics, vol. 121, pp. 331-354, 2000.

[7] T. McKelvey, H. Akcay, and L. Ljung, "Subspace-based multivariable system identification from frequency response data," IEEE Trans. Automatic Control, vol. 41, no. 7, pp. 960-979, 1996.

[8] A. Damen, P. Van den Hof, and A. Hajdasinksi, "Approximate realization based upon an alternative to the Hankel matrix: the Page matrix," Systems \& Control Letters, vol. 2, pp. 202-208, 1982.

[9] S. Van Huffel and J. Vandewalle, "Analysis and properties of the generalized total least squares problem $A X \approx B$ when some or all columns in $A$ are subject to error," Siam J. Matrix Analysis and Applications, vol. 10, no. 3, pp. 294-315, 1989.

[10] T. J. Abatzoglu, J. M. Mendel, and G. A. Harada, "The constrained total least squares technique and its applications to harmonic superresolution," IEEE Trans. Signal Processing, vol. 39, no. 5, pp. 1070-1087, 1991.

[11] M. Schuermans, P. Lemmerling, and S. Van Huffel, "Block-row Hankel weighted low rank approximation," Numerical Linear Algebra with Applications, vol. 13, pp. 293-302, 2006.

[12] M. Fazel, H. Hindi, and S. Boyd, "A rank minimization heuristic with application to minimum order system approximation," Proc. American Control Conf., pp. 4734-4739, 2001.

[13] C. L. Beck, J. C. Doyle, and K. Glover, "Model reduction of multidimensional and uncertain systems," IEEE Trans. Automatic Control, vol. 41, pp. 1466-1477, Jan. 1996.

[14] B. Recht, M. Fazel, and P. Parrilo, "Guaranteed Minimum-Rank Solutions of Linear Matrix Equations via Nuclear Norm Minimization," SIAM Review, vol. 52, no. 3, pp. 471-501, 2010.

[15] K. Mohan and M. Fazel, "Reweighted nuclear norm minimization with application to system identification," Proc. American Control Conference, pp. 2953-2959, 2010

[16] H. Hjalmarsson, J. S. Welsh, and C. R. Rojas, "Identification of Box-Jenkins models using structured ARX models and nuclear norm relaxation," Proc. American Control Conf., pp. 1-6, Apr. 2012.

[17] M. Ayazoglu and M. Sznaier, "An Algorithm for Fast Constrained Nuclear Norm Minimization and Applications to System Identification," in Proc. Control and Decision Conf., pp. 3469-3475, Dec. 2012.

[18] A. Hansson, Z. Liu, and L. Vandenberghe, "Subspace System Identification via Weighted Nuclear Norm Optimization," in Swedish Reglermote Conf., pp. 1-6, Feb. 2012.

[19] Z. Liu and L. Vandenberghe, "Interior-point method for nuclear norm approximation with application to system identification," Siam J. Matrix Analysis And Applications, vol. 31, no. 3, pp. 1235-1256, 2009.

[20] C. Grossmann, C. Jones, and M. Morari, "System identification via nuclear norm regularization for simulated moving bed processes from incomplete data sets," in Proc. IEEE Control and Decision Conf., pp. 4692-4697, 2009.

[21] T. Tanaka, C. Langbort, L.K. Mestha, and A.E. Gil, "Blind Source Separation by Nuclear Norm Minimization and Local Recoverability Analysis," IEEE Signal Processing Letters, vol. 20, no. 8, pp. 827-830, 2013.
[22] S. Boyd, N. Parikh, E. Chu, B. Peleato, and J. Eckstein, "Distributed Optimization and Statistical Learning via the Alternating Direction Method of Multipliers," Foundations and Trends in Machine Learning, vol. 3, no. 1, pp. 1-122, 2011

[23] F. Paganini, "A set-based approach for white noise modeling," IEEE Trans. Automatic Control, vol. 41, no. 10, pp. 1453-1465, 1996.

[24] R. S. Smith, "Nuclear norm minimization methods for frequency domain subspace identification," Proc. American Control Conf., pp. 2689-2694, June 2012.

[25] S. Venkatesh and M. Dahleh, "Identification in the presence of classes of unmodeled dynamics and noise," IEEE Trans. Automatic Control, vol. 42, no. 12, pp. 1620-1635, 1997.

[26] B. L. Ho and R. E. Kalman, "Effective construction of linear statevariable models from input/output functions," Regelungstechnik, vol. 12 , pp. 545-548, 1966.

[27] R. Gupta and F. Fairman, "Derivation of the Ho algorithm," IEEE Trans. Automatic Control, vol. 19, no. 3, pp. 270-271, 1974.

[28] A. J. Tether, "Construction of minimal linear state-variable models from finite input-output data," IEEE Trans. Automatic Control, vol. 15, no. 4 , pp. 427-436, 1970.

[29] M. Grant and S. Boyd, "CVX: Matlab software for disciplined convex programming, version 2.0 beta." http: //cvxr.com/cvx, Sept. 2012.

[30] M. Grant and S. Boyd, "Graph implementations for nonsmooth convex programs," in Recent Advances in Learning and Control (V. Blondel, S. Boyd, and H. Kimura, eds.), Lecture Notes in Control and Information Sciences, pp. 95-110, Springer-Verlag Limited, 2008. http://stanford.edu/ boyd/graph_dep.html.

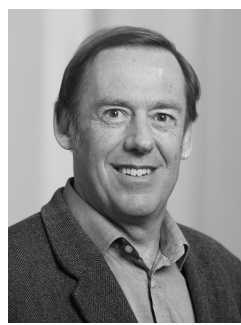

Roy Smith is a Professor of Electrical Engineering at the Swiss Federal Institute of Technology (ETH), Zürich. Prior to joining ETH in 2011 he was with the University of California, Santa Barbara, from 1990 to 2010. His Ph.D. is from the California Institute of Technology (1990) and his undergraduate degree is from the University of Canterbury (1980) in his native New Zealand. He has also been a long-time consultant to the NASA Jet Propulsion Laboratory, as well as having industrial experience in automotive control and power system design.

His research interests are mostly focused on the modeling, identification and control of uncertain systems. Particular control application domains of interest include chemical processes, flexible structure vibration, spacecraft and vehicle formations, semiconductor fabrication facilities, automotive engines, Mars aeromanoeuvring entry design, energy management in buildings and thermoacoustic machines. Dr. Smith is a Fellow of the IEEE, an Associate Fellow of the AIAA and a member of SIAM, AACZ and NZAC. 